Communications in Physics, Vol. 28, No. 1 (2018), pp. 61-73

DOI:10.15625/0868-3166/28/1/11084

\title{
INFLUENCE OF TEMPERATURE AND CONCENTRATION OF ETHANOL ON PROPERTIES OF BOROSILICATE GLASS PHOTONIC CRYSTAL FIBER INFILTRATED BY WATER - ETHANOL MIXTURE
}

\author{
HIEU LE VAN ${ }^{1,2, \dagger}$, HUE THI NGUYEN ${ }^{2}$, QUANG HO DINH ${ }^{3}$ AND VAN CAO LONG ${ }^{1}$ \\ ${ }^{1}$ Institute of Physics, University of Zielona Góra, Prof. Szafrana 4a, 65-516 Zielona Góra, Poland \\ ${ }^{2}$ Department of Physics, Hong Duc University, \\ 565 Quang Trung Street, Thanh Hoa City, Vietnam \\ ${ }^{3}$ School of Chemistry, Biology and Environment, Vinh University, \\ 182 Le Duan Street, Vinh City, Nghe An Province, Vietnam \\ ${ }^{\dagger}$ E-mail: hieulevan.ch17dhv@gmail.com
}

Received 16 January 2018

Accepted for publication 21 March 2018

Published 26 March 2018

\begin{abstract}
In this paper, we present a numerical simulation of the properties of a photonic crystal fiber (PCF) made of borosilicate glass infiltrated by the water-ethanol mixture. We examine the influence of temperature and ethanol concentration for the refractive index, dispersion properties, effective mode area and confinement loss of the fundamental mode by a Lumerical simulation method. We also calculate the fundamental mode of the fiber infiltrated with a water-ethanol mixture with the concentration range of ethanol from $0 \%$ to $100 \%$ in the temperature range from $10^{\circ} \mathrm{C}$ to boiling point of ethanol. The results show that all fibers infiltrated with water-ethanol mixture have flat dispersion characteristics in the infrared range above 1.32. The best flatness exists for pure ethanol. Furthermore, it is possible to shift the zero-dispersion wavelength and modify fundamental properties of PCFs by both temperature and concentration of ethanol. The results obtained are important because of that we not only use their reasonable parameters for the design and manufacture but also use them in nonlinear phenomena and nonlinear applications of fibers as supercontinuum generation.
\end{abstract}

Keywords: Photonic crystal fibers, dispersion, temperature, supercontinuum generation.

Classification numbers: 42.81.-I, 42.79.-e, 42.81.Wg.

(C)2018 Vietnam Academy of Science and Technology 


\section{INTRODUCTION}

The invention of Lasers in the early 1960s opened a new age in the field of optics. The appearance of Lasers has been significant in both fundamental and technological science because of their special properties, such as high intensity, monochromaticity, and ideal coherence. They are excellent tools for observing many nonlinear optical effects which created a new domain of optics, namely nonlinear optics. Furthermore, the many applications of lasers have created whole new areas of specialization in our civilization. An outstanding example of this is the discovery of optical fibers which change diametrically the world of telecommunication, in particular, the internet. As in every other domain in physics, fast development always leads to some kind of "crisis", when the intrinsic limits of the considered subject are achieved. In telecommunication, very high transmitted density data demands an exponential increase in the bandwidth of transmitted light which cannot be guaranteed by classical step-index fibers.

During the same period, the development of nonlinear optics and optical fibers introduced nonlinear optical fibers [1]. That means creating a more new model of optical fibers using photonic crystal claddings i.e. the dielectric matter with a periodic structure of the refractive index. This new kind of fibers was later called photonic crystal fibers (PCFs). Due to their special optical properties (like the dispersion which plays an essential role in light propagation), it is possible for PCFs to be tailored to a high degree of freedom. Moreover, they can be adjusted to the needs of the experiment. The appearance of PCFs in the late 1990s [2-6] is considered as a revolution comparable with the invention of the laser before. PCFs overcome many limitations intrinsic to step index fibers and create an ideal platform for observing new optical phenomena, in particular, so-called supercontinuum generation (SG) which has reported for the first time in [7]. The high degree of freedom in dispersion engineering of PCF allows SG to be performed optimally in the wide range of available pump sources, so one can use very short input pulses as well as high power continuous wave sources. SG in PCF was immediately applied in many fields as optical coherence tomography, spectroscopy, optical frequency metrology, in producing many sensors of special types [8-12]. One of the most potential applications of photonic crystals is the possibility of creating compact integrated optical devices with photons as the carriers of information, then the speed and bandwidth of advanced communication systems can be increased dramatically [13]. Recently, PCFs promise to become the next generation of ultra-low loss transmission fiber, they have been also applied in power deliveries and nonlinear optics [14-16].

In this work, we consider the properties of a regular lattice PCF made of borosilicate glass infiltrated by the water-ethanol mixture. The simple reason to choose water-ethanol mixture is that the water-ethanol mixture has a relatively high nonlinear refractive index in comparison to solids, which can make it easier to observe nonlinear phenomena. Additionally, it is nontoxic, ease of manipulation, and the most important, due to the fact that it presents a zero-dispersion wavelength in the range of facilitating supercontinuum generation, we take it as a subject of our consideration. Last but not least, the PCF is made of borosilicate glass that has a high nonlinear refractive index, and good rheological properties that allow for thermal processing of the glass without crystallization [17]. We focus our attempt to research the influence of temperature and concentration on the fundamental properties of PCFs such as guided modes, chromatic dispersion, confinement loss and effective mode area by a numerical simulation method. In the simulation, PCFs are designed which bases on labeled NL_33B2, was made of fused silica glass and successfully used in 
supercontinuum generation. We assume that the fiber infiltrated with the water-ethanol mixture. Obtained results also are very useful, for example in numerical simulation of supercontinuum generation in considered PCF models by solving the so-called Generalized Nonlinear Schrödinger Equation $[18,19]$. According to with our knowledge, we consider for the first time a numerical simulation of linear properties of borosilicate glass PCF infiltrated by the water-ethanol mixture.

The scheme of our paper looks as follows. In Sec. II we describe the design process of PCFs. The simulation procedure will be presented in Sec. III. By this procedure, we consider the influence of temperatures and concentrations on dispersion properties in Sec. IV, on the modal area in Sec. V and on losses in Sec. VI. Section VII contains our conclusions.

\section{DESIGN OF THE PCFs}

PCFs are defined as a kind of two-dimensional photonic crystals, which consist of a central defect region surrounded by multiple air-holes that run along the fiber length. PCFs are divided into two basic categories. The first one is index-guiding PCF, guiding light by total internal reflection between a solid core and a cladding region with multiple air-holes like standard fibers [20]. The second one uses a perfectly periodic structure exhibiting a photonic band-gap (PBG) effect at the operating wavelength to guide light in a low index core-region, which is also called PBG fibers [21]. The design of PCFs is very flexible. All of their properties are related to the proposed design, which is characterized by the pitch $(\Lambda)$ of the periodic array, the air-holes diameter $(\mathrm{d})$ and the number of air-holes rings around the core $(\mathrm{N})$ [22]. Additionally, the use of different materials opens up a new degree of freedom for the fiber design, including pure silica, air, liquids even gases etc. In particular, one of the methods to engineer the properties of the PCFs is infiltrating the holes with some liquids because of interesting reasons. For example, application of liquids with various refractive indexes allows for the modification of dispersion properties of the fiber without changing its geometrical parameters. It also can be further modified all curvature of fiber dispersion characteristics [23-26]. Depending on the refractive index of the liquid, the guiding effect of the fiber can possibly be changed from guiding based on modified total internal reflection, to guiding based on the photonic band gap effect. This leads to the fact that the optical characteristics of the fiber can also be changed by varying the temperature and concentration of liquids since the ordinary and extraordinary refractive indices of liquid crystals highly depend on temperature and concentration of liquids [27,28].

In this study, we will design the fiber structures by the finite element method which is widely used for analyzing the optical waveguide. The cross-section of the designed PCF is shown in Fig. 1. It consists of seven rings of air holes ordered in a hexagonal lattice, where the central hole is omitted. Besides that, one outer air hole is also omitted because of two reasons. The first, the PCFs are designed which bases on labeled NL_33B2. The omitting of one outer hole is caused by the mistake during fabrication process. In fact, we simulated this structure without omitting outer hole and obtained results are practically the same in both cases. This follows the fact that the influence of holes on parameter of PCF focuses on the first ring or second rings holes and it will decrease for the outer holes. The second, in near future, we will measure the SC generation for our designed model and will compare it with the related simulations. For this purpose, we need to keep its structure the same in simulation and experiment.

The core is solid and it made up of borosilicate glass. Dispersion properties are optimized by relative air-hole sizes in the first ring of the photonic cladding. The relative air hole size of 
the outer rings is decreased in order to increase the modal losses of the higher order modes. We verified this numerically, when the fiber guides only a few modes, while losses of higher order modes are significantly higher than the one for the fundamental mode.

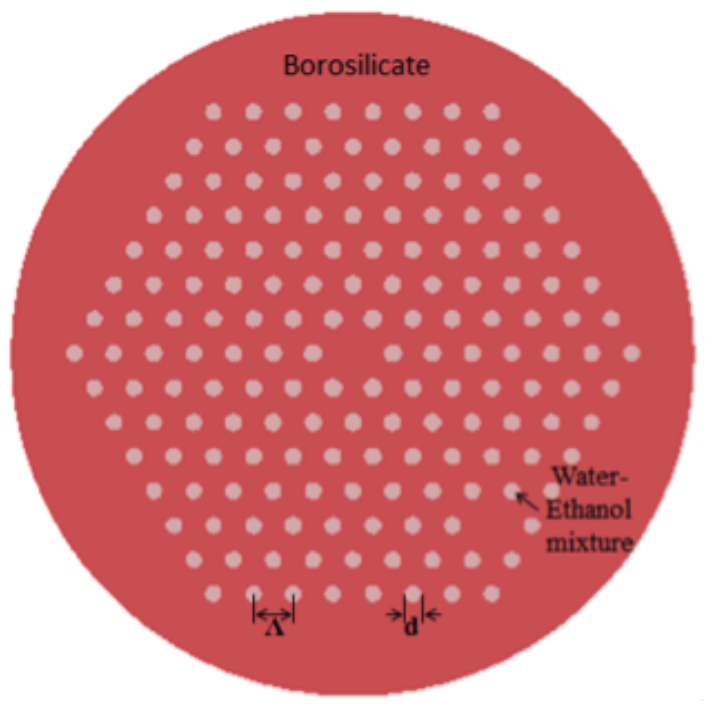

Fig. 1. Schematic cross-section diagram of PCFs with seven air hole rings and the geometrical parameters of the air hole diameter d, pitch $\Lambda$.

The fiber had a lattice constant of approximately $\Lambda=3.256$ and a filling factor $d / \Lambda=$ 0.457 on the first ring. For the remaining outer rings, the diameter of air holes varies between 1.35 and 1.488 then the filling factor varied in the range of $0.4146-0.457$ due to technological inaccuracies. The diameter of the core equals to 5.024. The geometrical parameters of the fiber are given in Table 1 .

Table 1. Geometrical parameters of PCF NL_33B2.

\begin{tabular}{|l|l|}
\hline Geometrical parameters & NL $\_$33B2 \\
\hline Number of air holes & 7 \\
\hline lattice constant $-\Lambda[\mu \mathrm{m}]$ & 3.256 \\
\hline core diameter $-\mathrm{d}_{\text {core }}[\mu \mathrm{m}]$ & 5.024 \\
\hline air hole diameter 1 st row $-\mathrm{d}_{1}[\mu \mathrm{m}]$ & 1.488 \\
\hline air hole diameter $2 \mathrm{nd}$ row $-\mathrm{d}_{2}[\mu \mathrm{m}]$ & 1.435 \\
\hline relative air-hole size 1 st row $-\mathrm{d}_{1} / \Lambda$ & 0.457 \\
\hline relative air-hole size 2 st row $-\mathrm{d}_{2} / \Lambda$ & 0.4407 \\
\hline
\end{tabular}

As it has been emphasized above, the PCF made of borosilicate glass demonstrates a high nonlinear refractive index, and good rheological properties which are suitable for thermal processing of the glass without crystallization. Its chemical composition is: $55 \% \mathrm{SiO}_{2}, 1 \% \mathrm{Al}_{2} \mathrm{O}_{3}, 26 \%$ 
$\mathrm{B}_{2} \mathrm{O}_{3}, 3 \% \mathrm{Li}_{2} \mathrm{O}, 9.5 \% \mathrm{Na}_{2} \mathrm{O}, 5.5 \% \mathrm{~K}_{2} \mathrm{O}$, and $0.8 \% \mathrm{As}_{2} \mathrm{O}_{3}[29]$. The $\mathrm{NC} 21$ glass refractive index is described using the following Sellmeier relation:

$$
n(\lambda)=\sqrt{1+\frac{B_{1} \lambda^{2}}{\lambda^{2}-C_{1}}+\frac{B_{2} \lambda^{2}}{\lambda^{2}-C_{2}}+\frac{B_{3} \lambda^{2}}{\lambda^{2}-C_{3}}}
$$

with coefficients: $B_{1}=1.15702228, B_{2}=0.14959764, B_{3}=1.36007514, C_{1}=0.00614152 \mu \mathrm{m}^{2}$, $C_{2}=0.02521981 \mu \mathrm{m}^{2}$, and $C_{3}=122.8441325 \mu \mathrm{m}^{2}$.

\section{NUMMERICAL SIMULATION}

In the simulations, by using Lumerical MODE Solutions software [30] we considered the influence of temperature and concentration of ethanol solution on the properties of the fundamental mode. The PCFs assumed that the air holes are filled with water-ethanol mixture. The refractive index of the water-ethanol mixture is a function of wavelength $\lambda$, temperature $t$ and volume concentration of ethanol c. It is given by the following formula [31]:

$$
n_{e w}(\lambda, t, c)=c n_{e}(\lambda, t)+(1-c) n_{w}(\lambda, t)
$$

where $c$ is the concentration of ethanol; $n_{e w}, n_{e}$ and $n_{w}$ denote refraction indices of the ethanolwater mixture, ethanol and water, respectively.

In the case of water, we have used the Cauchy formula with temperature dependent coefficients [32]

$$
n_{w}(\lambda, t)=A(t)+\frac{B(t)}{\lambda^{2}}+\frac{C(t)}{\lambda^{4}}+\frac{D(t)}{\lambda^{6}}
$$

where $\lambda$ is wavelength in $\mathrm{nm} ; t$ is the temperature in ${ }^{\circ} \mathrm{C}$ and $A(t), B(t), C(t), D(t)$ are Cauchy coefficients which are functions of temperature according to the following equations [33]:

$$
\begin{aligned}
& A(t)=1.3208-1.2325 .10^{-5} t-1.8674 .10^{-6} t^{2}+5.0233 .10^{-9} t^{3}, \\
& B(t)=5208.2413-0.5179 t-2.284 .10^{-2} t^{2}+6.9608 .10^{-5} t^{3} \\
& C(t)=-2.5551 .10^{8}-18341.336 t-917.2319 t^{2}+2.7729 t^{3}, \\
& D(t)=9.3495+1.7855 .10^{-3} t+3.6733 .10^{-5} t^{2}-1.2932 .10^{-7} t^{3},
\end{aligned}
$$

In the case of ethanol, dependence of refractive index $n_{e}$ on the temperature $t$ is taken from the formula [34]:

$$
n_{e}(\lambda, t)=\sqrt{1+\frac{B_{1} \lambda^{2}}{\lambda^{2}-C_{1}}+\frac{B_{2} \lambda^{2}}{\lambda^{2}-C_{2}}}-\alpha_{e}\left(t-T_{0}\right)
$$

where $T_{0}$ is $20^{\circ} \mathrm{C}$, temperature coefficient of ethanol $\alpha_{e}=3.94 \times 10^{-4} / \mathrm{K}$, and Sellmeier coefficients of ethanol: $B_{1}=0.83189, B_{2}=-0.15582, C_{1}=0.0093 \mu \mathrm{m}^{2}, C_{2}=-49.452 \mu \mathrm{m}^{2}$.

Finally, we obtain the following formula for refractive index of water-ethanol mixture:

$$
n_{e w}(\lambda, t, c)=c \cdot \sqrt{1+\frac{B_{1} \lambda^{2}}{\lambda^{2}-C_{1}}+\frac{B_{2} \lambda^{2}}{\lambda^{2}-C_{2}}}-\alpha_{e}\left(t-T_{0}\right)+(1-c)\left(A(t)+\frac{B(t)}{\lambda^{2}}+\frac{C(t)}{\lambda^{4}}+\frac{D(t)}{\lambda^{6}}\right) .
$$


Fig. 2 showed the intensity distribution of the fundamental mode at a wavelength of 1.55 $\mu \mathrm{m}$. Fig. 3 shows the examples of how the refractive index of the fiber filled with a water-ethanol mixture depends on the various wavelengths, temperatures, and volume fractions. The result shows that refractive index will increase with the increase of concentration and decrease when the temperature increases.

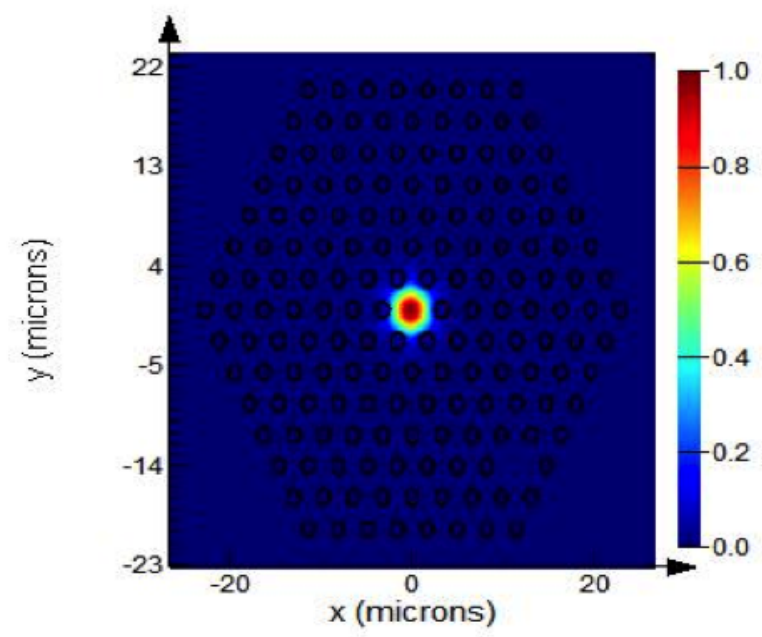

Fig. 2. Intensity distribution in the PCF filled water-ethanol mixture at $c=0.5$ and $T=30^{\circ} \mathrm{C}$. The color scale is in relative units.

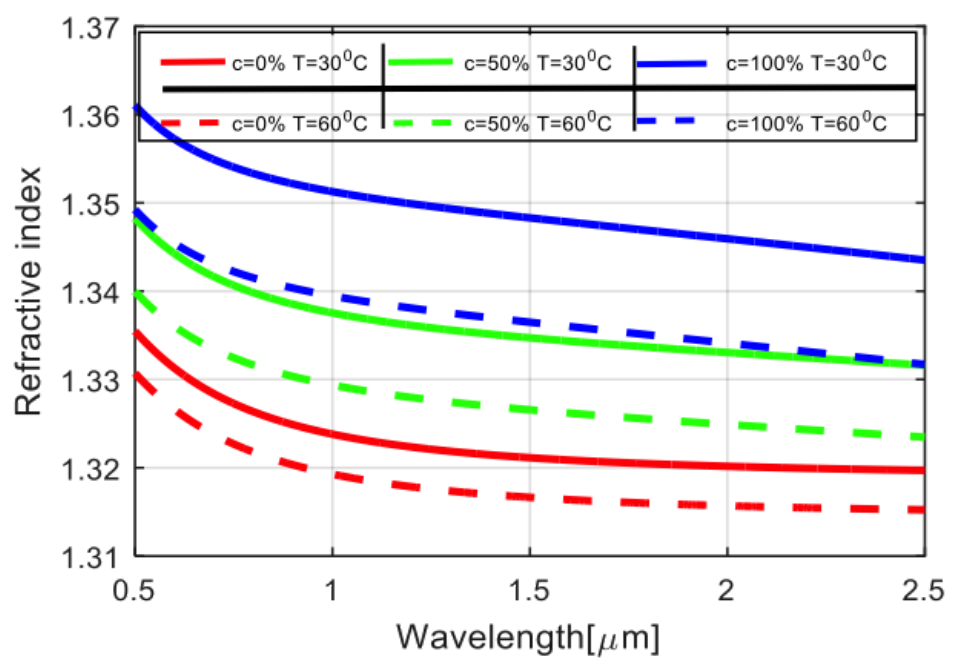

Fig. 3. Refractive index characteristic of the fiber filled with water- ethanol mixture with change of wavelength for various concentrations and temperatures. 


\section{INFLUENCE OF TEMPERATURES AND CONCENTRATIONS ON THE DISPERSION PROPERTIES}

We have calculated the dispersion of the fundamental mode as a function of the wavelength in the range of 0.5-2 for both the case of dry fibers and fibers filled by the water-ethanol mixture. In the simulation, the temperature of water-ethanol mixture changes in the range from $10^{\circ} \mathrm{C}$ to $70^{\circ} \mathrm{C}$ (boiling point) and the ethanol concentration changes between $0 \%$ and $100 \%$. The sample dispersion characteristics are plotted in Fig. 4.

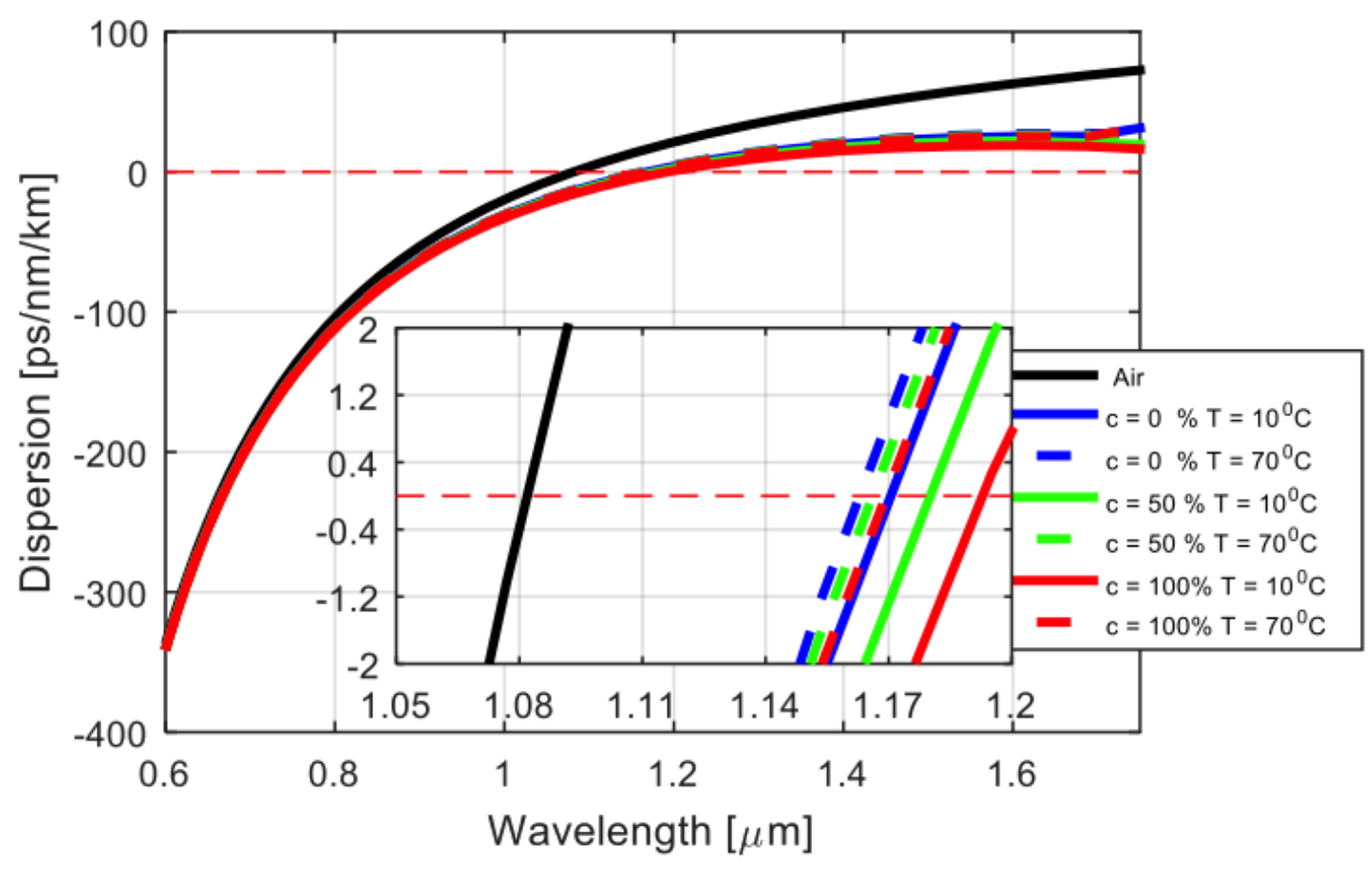

Fig. 4. Dispersion properties of the PCFs of structure NL_33B2 with various temperatures and concentrations of water- ethanol mixture.

The results showed that all fibers infiltrated with various water-ethanol mixture have flatted dispersion characteristics in the near-infrared range from 1.32 to 1.95 (the change is smaller than $10 \mathrm{ps} / \mathrm{nm} / \mathrm{km}$ ). The dispersion characteristics exist in both normal and anomalous dispersion regime. It is widely known that flat and near-zero dispersion plays a very important role in an efficiency of supercontinuum generation [35]. The zero dispersion wavelength (ZDW) for the dry fiber PCF is equal to 1.085. But when the holes are filled with water-ethanol mixture, the ZDW is shifted toward longer wavelengths, with a decreasing slope for the dispersion characteristic. It proved that the dispersion of properties PCF is better when air holes are filled with water-ethanol mixture.

The ZDW is also shifted towards longer wavelength the decreasing temperature and the increasing concentration of ethanol. In the case of ethanol concentration, this shift is in the range 
$0.007-0.0235$, which is smallest at $0 \%$ ethanol and biggest at $100 \%$ ethanol when temperature changes from $10^{\circ} \mathrm{C}$ to $70^{\circ} \mathrm{C}$. It can be seen from formula (9) which demonstrates the dependence of refractive index on temperature and concentration of ethanol in water that the refractive index will increase with the increase of concentration and decrease when the temperature increases. Besides that, one can explain this fact in a simple way such as increasing ethanol concentration leads to increase the density of molecules, consequently, increasing the interaction and collision between light and molecules. In other words, this leads to the fact that speed of light propagation decreases, while the density of molecules in a liquid usually decreases corresponding to the increase of temperature; it is not surprising that the speed of light in a liquid will normally increase when the temperature increases. Thus, the index of refraction normally decreases when the temperature of a liquid increases. This has been proven in many theoretical and experimental works. The shifts of the ZDW with the temperature and ethanol concentration are summarized in Table 2 and which has been presented in Fig. 5.

Table 2. The value of ZDW with various temperatures and various concentrations of ethanol.

\begin{tabular}{|l|ccccccc|}
\hline $\mathrm{C} \backslash \mathrm{T}$ & 10 & 20 & 30 & 40 & 50 & 60 & 70 \\
\hline 0 & 1.1694 & 1.1688 & 1.168 & 1.167 & 1.1655 & 1.1644 & 1.1628 \\
\hline 0.1 & 1.1713 & 1.1705 & 1.1693 & 1.1682 & 1.1668 & 1.1652 & 1.1635 \\
\hline 0.2 & 1.1735 & 1.1722 & 1.171 & 1.1695 & 1.1678 & 1.1662 & 1.1642 \\
\hline 0.3 & 1.1756 & 1.1742 & 1.1725 & 1.1706 & 1.1688 & 1.1669 & 1.1648 \\
\hline 0.4 & 1.1778 & 1.1759 & 1.174 & 1.17109 & 1.1696 & 1.1675 & 1.1654 \\
\hline 0.5 & 1.18 & 1.1779 & 1.1762 & 1.1732 & 1.1708 & 1.1683 & 1.166 \\
\hline 0.6 & 1.182 & 1.1792 & 1.1765 & 1.1739 & 1.1713 & 1.1689 & 1.1665 \\
\hline 0.7 & 1.1849 & 1.1815 & 1.1788 & 1.1757 & 1.173 & 1.17 & 1.1672 \\
\hline 0.8 & 1.1875 & 1.184 & 1.1805 & 1.177 & 1.174 & 1.171 & 1.168 \\
\hline 0.9 & 1.1902 & 1.186 & 1.1825 & 1.1785 & 1.1752 & 1.1718 & 1.1688 \\
\hline 1.0 & 1.193 & 1.189 & 1.184 & 1.18 & 1.1763 & 1.1728 & 1.1695 \\
\hline & $\mathrm{ZDW}$ & $\mathrm{ZDW}$ & $\mathrm{ZDW}$ & $\mathrm{ZDW}$ & $\mathrm{ZDW}$ & $\mathrm{ZDW}$ & $\mathrm{ZDW}$ \\
\hline
\end{tabular}




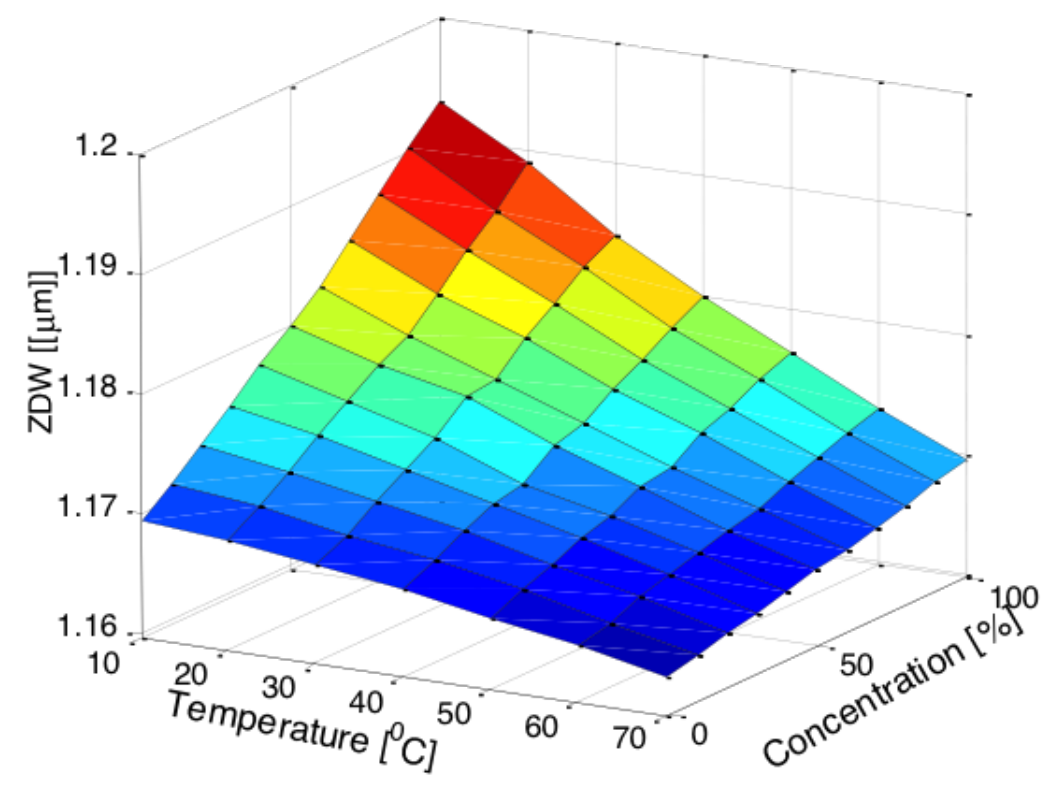

Fig. 5. The ZDW shift with various temperatures and ethanol concentrations.

The dependence of ZDW shift with various temperatures and ethanol concentrations can be defined approximately by second-degree polynomial:

$$
Z D W(c, t)=a_{00}+a_{10} \cdot c+a_{01} \cdot t+a_{20} \cdot c^{2}+a_{11} \cdot c \cdot t+a_{02} \cdot t^{2}
$$

where: $a_{00}=1.166, \mathrm{a}_{10}=-1.043 \mathrm{e}^{-4}, \mathrm{a}_{01}=2.785 \mathrm{e}^{-4}, \mathrm{a}_{20}=8.977 \mathrm{e}^{-08}, \mathrm{a}_{11}=-2.719 \mathrm{e}^{-06}, \mathrm{a}_{02}=$ $3.451 \mathrm{e}^{-07}$.

The shift plays an important role in supercontinuum generation (SG) because we generate supercontinuum basing on a match between the ZDW and fiber lasers which is controlled by the shifts. In particular, when the pump wavelength is near the ZDW on the anomalous dispersion side, the SG process becomes the most effective [36-38].

\section{INFLUENCE OF TEMPERATURES AND CONCENTRATIONS ON MODEL AREA}

The modal area is a quantity of a great importance because it was originally introduced as a measure of nonlinearities in the optical fiber. Therefore the modal area is a factor that determines the effective nonlinear medium for SG. In a simulation, we have calculated the modal area of the fundamental mode as a function of the wavelength in the range of $0.6-1.6$. The modal area characteristics are plotted in Fig. 6 and Fig. 7. The results show that modal area depends on the temperature and concentration of liquids. When the temperature increases, mode area decreases. But this decreasing also depends on wavelength. The smallest decrease is observed at 0.6 (from 14.3919 to 14.251 , this is $0.98 \%$ ) and the biggest at 1.6 (from 23. 166 to 21.646, this is $6.56 \%$ ). It means that as the wavelength increases, its decrease also increases. For the case of 
ethanol concentration, when the concentration of ethanol increases, the mode area also increases. In addition, it is clear that the increase in the mode area of each case is relatively small, which is beneficial for nonlinear applications.

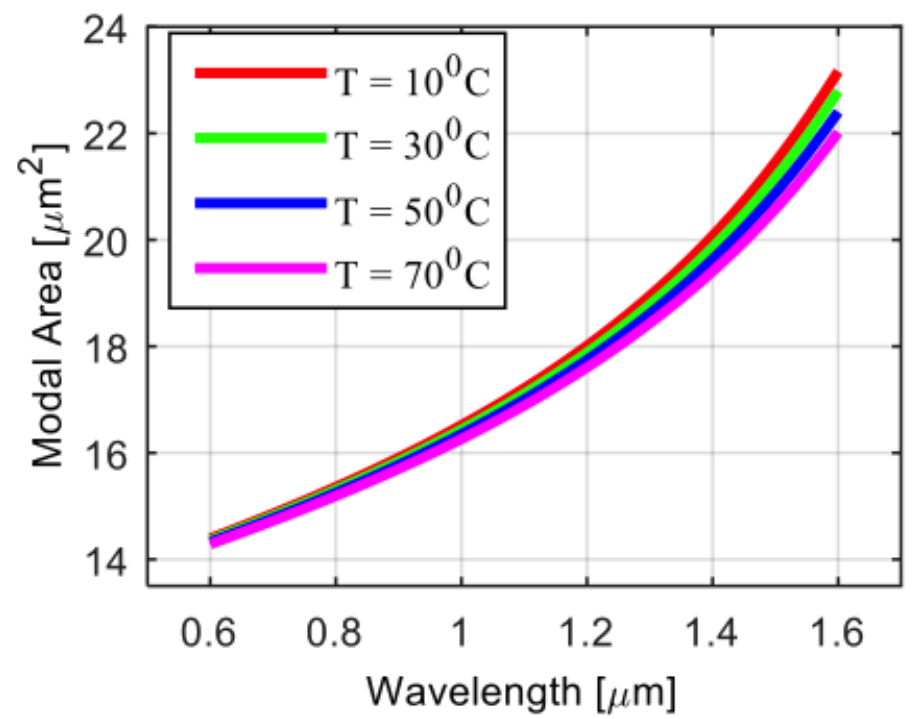

Fig. 6. Mode area of the fundamental mode for the fiber with various temperatures at $\mathrm{c}=0.5$.

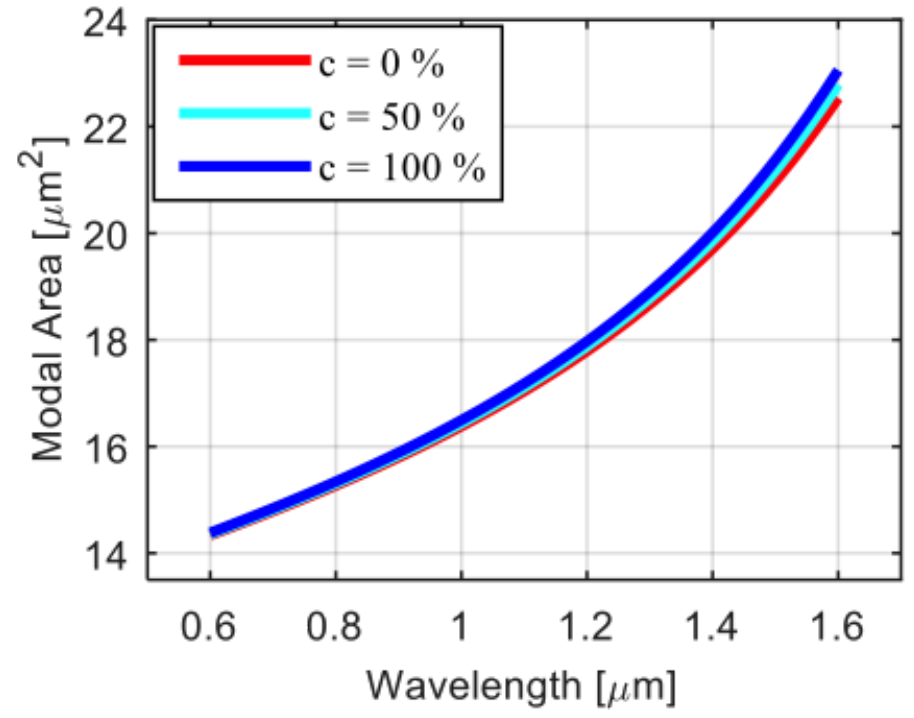

Fig. 7. Mode area of the fundamental mode for the fiber with various concentrations at $T=30^{\circ} \mathrm{C}$. 


\section{INFLUENCE OF TEMPERATURES AND CONCENTRATIONS ON CONFINEMENT LOSS}

We have calculated the attenuation of the fibers as a function of wavelength for fiber filled with water-ethanol mixture at various temperatures and concentrations of ethanol. In fact, the core is all solid and we only consider the fundamental mode. The results are plotted in Figures 8 and 9.

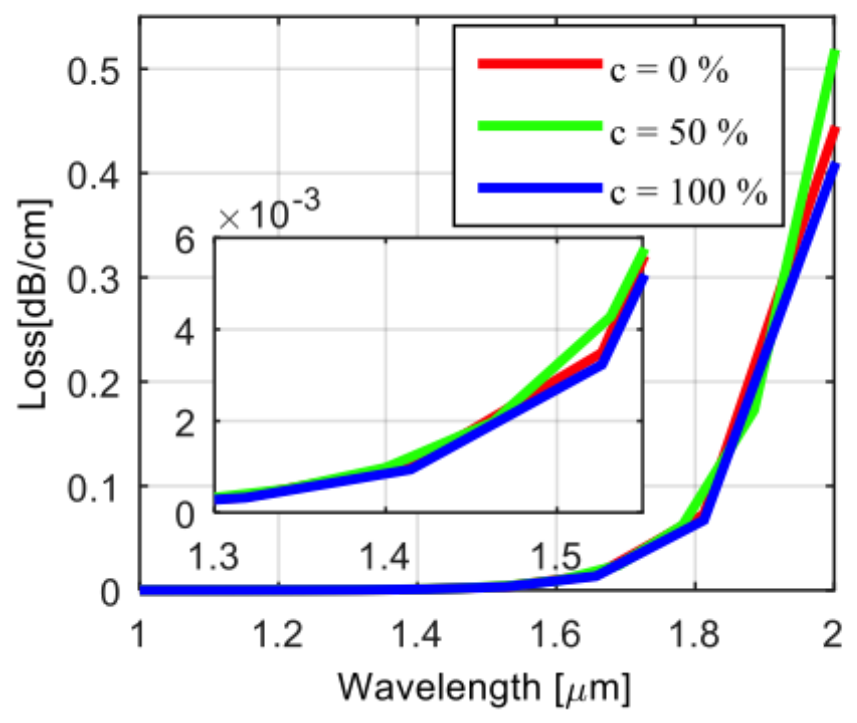

Fig. 8. Confinement loss as a function of wavelength with various concentrations at $T=30^{\circ} \mathrm{C}$.

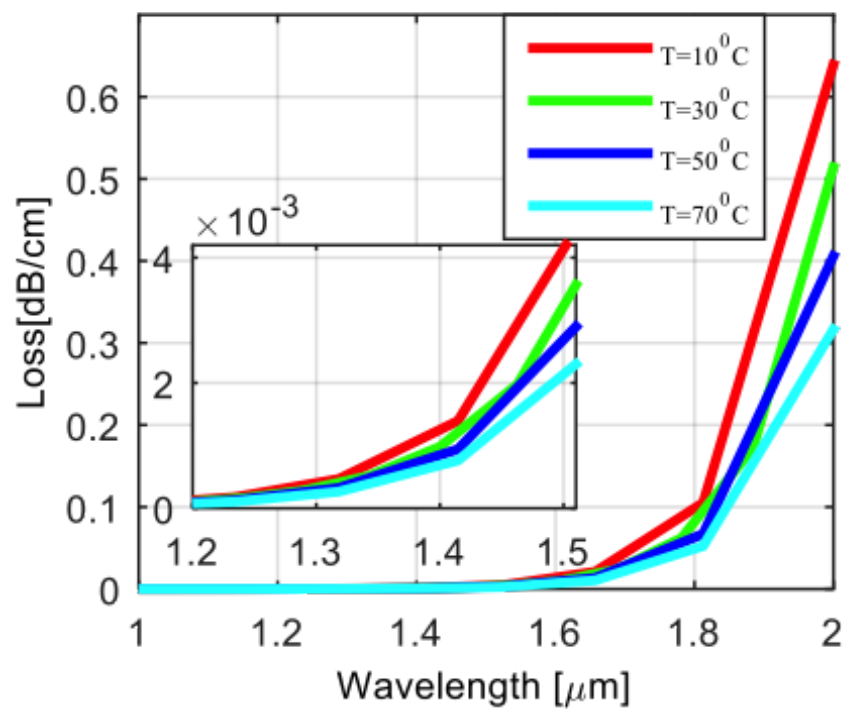

Fig. 9. Confinement loss as a function of wavelength with various temperatures at $c=0.5$. 
HIEU LE VAN, HUE THI NGUYEN, QUANG HO DINH AND VAN CAO LONG

The results show that the losses maintain an overall tendency to increase with increasing wavelength. Besides that, the losses also depend on the temperatures and concentrations of ethanol. The lowest losses are observed for fiber infiltrated with water and highest losses are observed for ethanol. It means that the losses increase with increasing concentration of ethanol. This is totally consistent in practice because infiltration of loss liquids can introduce significant loss in transmission. In the case of the change of temperature, the results show that with increasing temperature the loss decreases. This is understandable because the temperature will change the population of different states of the molecule, particularly the rotational states which will affect the intensity of the absorption lines.

\section{CONCLUSION}

In this paper, we presented a numerical study of the properties of nonlinear photonic crystal fibers filled by water-ethanol mixtures. We analyzed PCFs which made of borosilicate glass consisting seven rings of air holes ordered in a hexagonal lattice with a diameter of the core equals to 5.024. The results showed that we are able to control the shape and the ZDW of the dispersion characteristic of the water-ethanol mixtures filled PCF by changing the temperatures or ethanol concentrations. Greater changes of the ZDW can be obtained for higher concentrations of ethanol and for lower temperatures. The reason is that the refractive index of water-ethanol mixtures depends on temperature and concentration of ethanol. Consequently, the changes in temperature and concentration of ethanol also change properties of the fiber. The cause of this change is that when the temperature and concentration change, the density of water-ethanol mixture changes. Additionally, at the different temperatures, the refractive index of the water-ethanol mixtures also changes much more than those of the glasses. These results are pretty important because of that we not only use their reasonable parameters for the design and manufacture but also use them in nonlinear phenomena and nonlinear applications of fibers. In particular, we will use them in numerical simulation for supercontinuum generation in some considered models of PCFs by solving the so-called Generalized Nonlinear Schrödinger Equation. This will be the subject of our future publication.

\section{ACKNOWLEDGEMENTS}

I would like to express our gratitude Prof. Ryszard Buczyñski and Prof. Rafal Kasztelanic for useful discussions.

\section{REFERENCES}

[1] G. P. Agrawal, Nonlinear Fiber Optics, Fifth edition, Elsevier 2013.

[2] J. C. Knight et. al. Optics Letters 21 (19) (1996) 1547-1549.

[3] J.C. Knight, J. Broeng, T.A. Birks, P.St.J. Russell, Science 282 (5393) (1998) 1476-1478.

[4] P. S. J. Russell, Journal of light wave technology 24 (12) (2006) 4729-4749.

[5] P. Russell, Science 299 (5605) (2003) 358-362.

[6] J. K. Ranka et. al. Optics Letters 25 (11) (2000) 796-798.

[7] Wadsworth, W. J., J. C. Knight, W. H. Reewes, P. S. J. Russell, and J. Arriaga, Eletron. Lett.36 (17) (2000) 1452-1253.

[8] X. Liu, X. Zhou, X. Tang, J. Ng, J. Hao, T. Chai, E. Leong, C. Lu, IEEE Photon. Technol. Lett.17 (8) (2005) 1626-1628.

[9] N. Muduli, S.K. Tripathy, Optik 125 (16) (2014) 4363-4366. 
INFLUENCE OF TEMPERATURE AND CONCENTRATION OF ETHANOL ON PROPERTIES OF BOROSILICATE ... 73

[10] J.M.Dudley, G. Genty, S. Coen, Rev. Mod. Phys. 78 (4) (2006) 1135-1178.

[11] R.Wang, J.Yao, Y.Miao, Y.Lu, D.Xu, N.Luan, M.Musideke, L. Duan, C. Hao, Sensors 13 (6) (2013) 7916-7925.

[12] G.P.Agrawal, Fiber optic communication systems, $3^{\text {nd }} e d$. Wiley, ISBN 0-471-22114-7 (Electronic), 2002.

[13] D. Chen, Laser Phys.Lett. 4 (6) (2007) 437-439.

[14] N. G. R. Broderick, T. M. Monro, P. J. Bennett, D. J. Richardson, Opt. Lett. 24 (20) (1999) 1395-1397.

[15] J. M. Dudley, J. R. Taylor, Nature Photonics 3 (2) (2009) 85-90.

[16] D. Lorenc, M. Aranyosiova, R. Buczynski, R. Stepien, I. Bugar, A. Vincze, and D. Velic, Appl. Phys. B 93 (2008) 531-538.

[17] D. Pysz, I. Kujawa, R. Stepien, M. Klimczak, A. Filipkowski, M. Franczyk, L. Kociszewski, J. Buniak, K. Harany, R. Buczynski, Bull. Pol. Acad. Sci. Tech. Sci. 62 (4) (2014), 667-682.

[18] Cao Long Van, Communications in Physics 26 (4) (2016) 301-323.

[19] J. C. Knight, T. A. Birks, P. S. J. Russell, D. M. Atkin, Opt. Lett. 21 (19) (1996) 1547-1549.

[20] T. A. Birks, J. C. Knight, P. St. J. Russell, Opt. Lett. 22 (13) (1997) 961-963.

[21] S. A. Cerqueira Jr, Rep. Prog. Phys. 73 (2) (2010) 024401.

[22] J. C. Knight, T. A. Birks, P. St. J. Russell, J. P. de Sandro, J. Opt. Soc. Am. A 15 (3) (1998) 748-752.

[23] W. Lin, Y. Miao, B. Song, H. Zhang, B. Liu, Y. Liu, D. Yan, Opt. Comm. 336 (2015) 14-19.

[24] P.S. Maji, P.R. Chaudhuri, Optik 125 (20) (2014) 5986-5992.

[25] J. Pniewski, T.Stefaniuk, H. Le Van, V. Cao Long, L. Chu Van, R. Kasztelanic, G. Pniewski, A. Ramaniuk, M. Trippenbach, R. Buczynski, Applied Optics 55 (19) (2016) 5033-5040.

[26] E. Yoshida, A.Wada, N. Karasawa, Japanese Journal of Applied Physics 55 (7) (2016) 072501.

[27] Hieu Le Van, R. Buczynski, V.C. Long, M.Trippenbach, K. Borzycki, An Manh Nguyen, R. Kasztelanic, Optics Communications 407 (2017), 417-422.

[28] N. Karasawa, Appl. Opt. 51 (21) (2012) 5259-5265.

[29] R. Buczynski, D. Pysz, R. Stepien, R. Kasztelanic, I. Kujawa, M. Franczyk, A. Filipkowski, Andrew J.Waddie , M. R. Taghizadeh, Journal of the European optical society-Rapid publications 6 (2011) 11038.

[30] Lumerical Solutions Inc., http://www.lumerical.com

[31] L. Chu Van, T. Stefaniuk, R. Kasztelanic, V. Cao Long, M. Klimczak, H. Le Van, M. Trippenbach, R.Buczyski, Proc. of SPIE 9816 (2015) 981600-1.

[32] A. Bashkatov, E. Genina, Proc. of SPIE 5068 (2003).

[33] J. Lei, S. Hou, Y. Liu, X. Li, Progress In Electromagnetics Research Symposium Proceedings (2014) 565-568.

[34] G. Stepniewski, R. Kasztelanic, D. Pysz, R. Stepien, M. Klimczak, R. Buczynski, Optical Materials Express 6 (8) (2016) 2689-2703.

[35] A. Ferrando, E. Silvestre, P. Andrés, J. J. Miret, and M. V. Andrés, Optics Express 9 (2001) 687-697.

[36] F. Begum, Y. Namihira, T. Kinjo, S. Kaijage, Optics Communications 284 (2011) 965-970.

[37] X. Luo, J. Peng, L. Cheng, L. Yang, N. Dai, H. Li,and J. Li, IEEE Photonics Journal 7 (2015) 4501309.

[38] H. Saghaei, M.K. Moravvej-Farshi, M. Ebnali-Heidari, M.N. Moghadasi, IEEE Journal of Selected Topics in Quantum Electronics 22 (2016) 4900508. 